\title{
Magnus force and acoustic Stewart-Tolman effect in type II superconductors
}

\author{
V.D. Fil ${ }^{1}$, D. V.Fil ${ }^{2}$, A. N. Zholobenko ${ }^{1}$, N. G. Burma ${ }^{1}$, Yu. A. Avramenko ${ }^{1}$, J. D. Kim ${ }^{3}$, S. M. Choi ${ }^{3}$, and S. I. Lee ${ }^{3,4}$ \\ ${ }^{1}$ B. Verkin Institute for Low Temperature Physics and Engineering, \\ National Academy of Sciences of Ukraine, Lenin av. 47 Kharkov 61103, Ukraine \\ ${ }^{2}$ Institute for Single Crystals, National Academy of Sciences of Ukraine, Lenin av. 60, Kharkov 61001, Ukraine \\ ${ }^{3}$ Pohang University of Science and Technology, Pohang, 794784, Korea \\ ${ }^{4}$ Korea Basic Science Institute, Daejeon, 305333, Korea
}

\begin{abstract}
At zero magnetic field we have observed an electromagnetic radiation from superconductors subjected by a transverse elastic wave. This radiation has an inertial origin, and is a manifestation of the acoustic Stewart-Tolman effect. The effect is used for implementing a method of measurement of an effective Magnus force in type II superconductors. The method does not require the flux flow regime and allows to investigate this force for almost the whole range of the existence of the mixed state. We have studied behavior of the gyroscopic force in nonmagnetic borocarbides and $\mathrm{Nb}$. It is found that in borocarbides the sign of the gyroscopic force in the mixed state is the same as in the normal state, and its value (counted for one vortex of unit length) has only a weak dependence on the magnetic field. In $\mathrm{Nb}$ the change of sign of the gyroscopic force under the transition from the normal to the mixed state is observed.
\end{abstract}

PACS numbers: 74.25.Qt, 74.70.Ad

The Stewart-Tolman (ST) effect, the emergence of an electrical current in a coil under an accelerate or decelerate rotation, is a famous experimental proof for the electron nature of conductivity of metals. The inertial force should give a contribution to the electrical currents excited by a transverse acoustic wave propagating in the metal (the acoustic ST effect). Nevertheless, it was believed that this effect is negligible and does not have any practical use. As far as we know, up to now there was no efforts to register inertial force in acoustic experiments. In our study we have observed the acoustic ST effect and use it for a development of a powerful method of investigation of the gyroscopic forces acting on a moving vortex in type II superconductors.

Our study was motivated by the present situation in understanding of the nature of the gyroscopic force (the effective Magnus force [1]) in superconductors. Most of all, it concerns the so called Hall anomaly - the change of sign of the Hall voltage at the superconducting transition. According to current theoretical conceptions, this effect may have both the microscopic origin, connected with peculiarities of the electron spectrum [2], and the macroscopic one: the appearance of the transverse force under large (much large than the core size) displacements of the vortices in the pinning potential [3]. The flux flow experiments did not allow to separate these two contributions. The macroscopic effects may remove the genuine (microscopic) Hall anomaly or, on the contrary, mimic it. For instance, in $\mathrm{Nb}$ the Hall anomaly was observed or not, depending on the sample quality [4].

To overcome this difficulty we provide measurements of the Magnus force under an oscillatory motion of the vortices with a small (of the atomic scale) amplitude of the displacements. It allows to exclude the influence of the pinning forces. Another advantage of the method is the ability to fulfill measurements in almost the whole

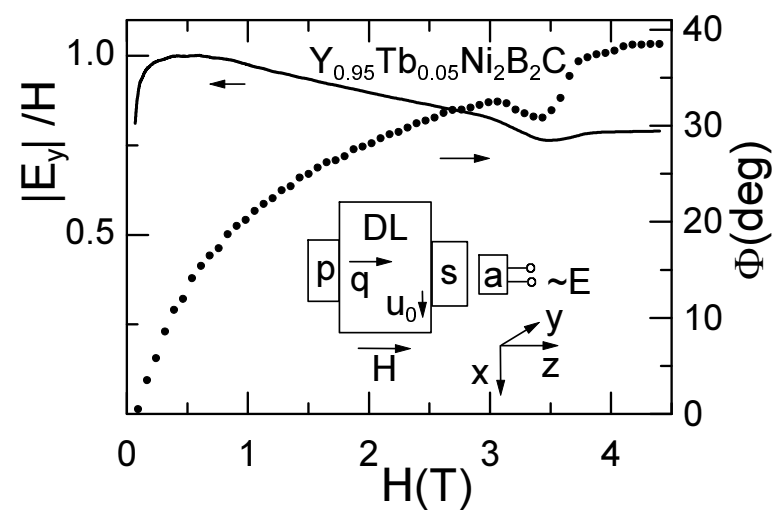

FIG. 1: The modulus (solid line) and the phase (dots) of $E_{y}(H)$ in $\mathrm{Y}_{0.95} \mathrm{~Tb}_{0.05} \mathrm{Ni}_{2} \mathrm{~B}_{2} \mathrm{C}$ at $T=1.7 \mathrm{~K}\left(H_{c 2}=3.8 \mathrm{~T}\right)$. The ratio $\left|E_{y}\right| / H$ is normalized as described in the text. Inset - the sketch of the experiment ( $\mathrm{p}$, the piezotransducer, DL, the delay line, s, the sample, and a, the antenna)

range of the existence of the mixed state.

The method is based on the measurements of the amplitude and the phase of an electromagnetic field (EF) radiated from the superconductor due to the vortex oscillations excited by a transverse elastic wave. The scheme of the experiment and the frame of reference are shown in Fig.1(inset). Technically, the method is close to the one used in [5]. A key new point is the registration not only large $E_{y}$, but also small $E_{x}$ component of the EF field. The $E_{x}$ component in the Meissner state is caused solely by the acoustic ST effect.

In our study we used the single crystals of nonmagnetic borocarbides $\left(\mathrm{YNi}_{2} \mathrm{~B}_{2} \mathrm{C}, \mathrm{Y}_{0.95} \mathrm{~Tb}_{0.05} \mathrm{Ni}_{2} \mathrm{~B}_{2} \mathrm{C}\right.$ and $\mathrm{LuNi}_{2} \mathrm{~B}_{2} \mathrm{C}$ ) and $\mathrm{Nb}$ as the objects of the investiga- 
tions. The borocarbide single crystals were prepared by the same method as in [6]. The samples have the shape of platelets with the thickness $\sim 0.5 \mathrm{~mm}$ and the transverse size $\sim 3 \mathrm{~mm}$. In all samples the $\mathrm{C}_{4}$ axis was oriented orthogonal to the platelet plane, which was used as the radiated surface. The working frequencies are $54 \div 55 \mathrm{MHz}$, and the intensity of the exciting signal is $\sim 10 \mathrm{~W} / \mathrm{cm}^{2}$. The details of the measuring technics are given in 7].

In the normal state the $E_{x}$ and $E_{y}$ components can be found from the joint solution of the Maxwell equations and the kinetic equation. With accounting for the gyroscopic forces this problem was solved in [8]. Let the vector of the elastic displacements is aligned along the $x$ axis $\left(\mathbf{u}(z, t)=\left(u_{0} \cos (q z) e^{i \omega t}, 0,0\right)\right)$. In the local limit ( $q l \ll 1, q$ is the wave number, and $l$ is the mean free path) and for $|\Omega| \tau \ll 1(\Omega$ is the cyclotron frequency, and $\tau$ is the relaxation time) the electrical field radiated from the sample at $z=0$ has the form:

$$
\begin{aligned}
& E_{x}^{(n)}=\left[1-i \beta_{d}\right] X u_{S T}+[1-X] X \Omega \tau u_{i n d} \\
& E_{y}^{(n)}=X u_{i n d}
\end{aligned}
$$

where $X=k_{n}^{2} /\left(1+k_{n}^{2}\right)$ with the dimensionless parameter $k_{n}^{2}=4 \pi i \omega \sigma_{0} / q^{2} c^{2}\left(\sigma_{0}=n e^{2} \tau / m\right.$, the static conductivity), $u_{S T}$ and $u_{\text {ind }}$ are the extraneous forces: $u_{S T}=m_{e} \omega^{2} u_{0} /|e|$ is the ST inertial force $\left(m_{e}\right.$ and $e$ are the mass and the charge of free electron, respectively) and $u_{\text {ind }}=i \omega H u_{0} / c$ is the inductive force, $H$ is the external magnetic field. The sign of charge of the carriers is included into the definition of $\Omega$. The term in Eq.(1) $\propto i \beta_{d}$ is caused by the deformation interaction. For the quadratic spectrum of the carriers $\beta_{d}=(1 / 5)\left(v_{F} / s\right) q l$, where $v_{F}$ is the Fermi velocity, and $s$ is the sound velocity. For the magnetic field used in the experiment $\left|u_{\text {ind }} / u_{S T}\right|=\Omega / \omega \gg 1$. This strong inequality makes the magnitude of the second (gyroscopic) term in Eq. (11) comparable or larger than that of the ST term, and allows to neglect the term $\propto u_{S T}$ in Eq. (2).

In the mixed state the EF can be evaluated from the following system of equations

$$
\begin{aligned}
& \frac{d^{2} \mathbf{E}}{d z^{2}}=\frac{4 \pi i \omega}{c^{2}} \mathbf{j}, \\
& \mathbf{j}=\sigma\left(\mathbf{E}+\mathbf{u}_{S T}+\frac{i \omega}{c}[\mathbf{w} \times \mathbf{B}]\right), \\
& \frac{1}{c}[\mathbf{j} \times \mathbf{B}]+\left(i \omega \eta+\alpha_{L}\right)(\mathbf{u}-\mathbf{w}) \\
& +i \omega \alpha_{M}\left[(\mathbf{u}-\mathbf{w}) \times \frac{\mathbf{B}}{|B|}\right]=0,
\end{aligned}
$$

where $B$ is the magnetic induction, $\mathbf{j}$ is the current, $\mathbf{w}$, the displacement of the vortex lattice, $\sigma=n_{s} e^{2} / i \omega m$, the a.c. conductivity in the superconducting state $\left(n_{s}\right.$ is the superfluid density), the dynamical parameters $\eta$, $\alpha_{L}, \alpha_{M}$, are the viscosity, the Labush parameter, and the Magnus coefficient, correspondingly. Eq. (3) is the Maxwell equation, Eq. (44) is the matter equation that connects the current with the field $\mathbf{E}$ and the extraneous forces, and Eq. (57) is the Galilean invariant equation of motion of the vortex lattice, normalized to the unit volume [9]. The boundary condition for the system (3)(5]) is $d \mathbf{E} /\left.d z\right|_{z=0} \approx 0$ [8].

Taking into account that for typical superconductors $\alpha_{M} \ll \eta$ and $q^{2} \lambda_{L}^{2} \ll 1\left(\lambda_{L}\right.$ is the London penetration depth) we obtain

$$
\begin{aligned}
& E_{x}^{(m)}=\left[1-i \beta_{d m}(B)\right] X(B) u_{S T}+ \\
& {[1-X(B)] X(B)\left[\frac{i \omega \alpha_{M}}{i \omega \eta+\alpha_{L}}\right] u_{i n d},} \\
& E_{y}^{(m)}=X(B) u_{i n d} .
\end{aligned}
$$

Here $u_{i n d}=i \omega B u_{0} / c$, and $X(B)=k_{m}^{2} /\left(1+k_{m}^{2}\right)$ with $k_{m}^{2}=4 \pi\left(i \omega \eta+\alpha_{L}\right) /\left(q^{2} B^{2}\right)$. The deformation interaction is not included into Eqs. (3)-(5). But since the structure of Eqs. (6), (7) is similar to one of Eqs. (1), (2), we add the deformation correction into Eq. (6) phenomenologically. One can assume that the quantity $\beta_{d m}$ is approximated as $\beta_{d m}(B) \approx \beta_{d} B / H_{c 2}$. Going ahead, we emphasize that for the measurement of $\alpha_{M}$ we do not need the exact form of $\beta_{d m}(B)$

The experimental procedure consists in the measuring of the relative changes of the fields $E_{x}$ and $E_{y}$ under a variation of $H$. To obtain the dynamical parameters from the experimental data one should set the reference points for the amplitude and the phase of $E_{x}$ and $E_{y}$. In other words, at certain $H$ we should determine the relation between $E_{x}, E_{y}$ and $u_{0}$.

According to the Bardin-Stephen estimate the viscosity $\eta \propto B$. Therefore, at $\mathrm{H}$ close to $H_{c 1}$ the quantity $\left|k_{m}^{2}\right| \gg 1$, and the modulus and the phase of $X$ approaches to 1 and 0, correspondingly. Let us count the phase of $E_{y}$ from its value at $H \rightarrow H_{c 1}$. Then $\arg E_{y}=\arg X$. On the other hand, at $H=H_{c 2}$ the quantity $\left|X\left(H_{c 2}\right)\right|=\left[1+\tan ^{2} \arg X\left(H_{c 2}\right)\right]^{-1 / 2}$ and it is convenient to choose such units for $\left|E_{y}\right|$ that satisfy the relation $\left|E_{y}\left(H_{c 2}\right)\right| / H_{c 2}=\left|X\left(H_{c 2}\right)\right|$.

If the Ginzburg-Landau parameter $\kappa \gg 1$, one can neglect the difference between $B$ and $H$ in almost the whole range of $H$ where the mixed state exists. Then, at $H \sim(5 \div 10) H_{c 1}$ (in which $\left|k_{m}^{2}\right| \gg 1$ ) the quantity $\left|E_{y}(H)\right| / H=|X(H)|$ (in the units of $\left|E_{y}\right|$ chosen) should approach unity. The fulfillment of this condition can be considered as an independent test for the measuring procedure proposed. If this condition fails it can be due to a strong non-uniformity of the conductivity near the surface of the sample (see [ $[$ ), and in this case one cannot use the relations (6), (7) for the analysis of the results of the measurements. In the experiments presented in this paper the above mentioned condition was fulfilled with the accuracy $\sim 5 \%$. The typical example of the experimental data is presented in Fig. [1 From these data one can find the dependencies $\eta(H)$ and $\alpha_{L}(H)[10$.

At $H=0$ and $T \ll T_{c}$ the deformation correction is 


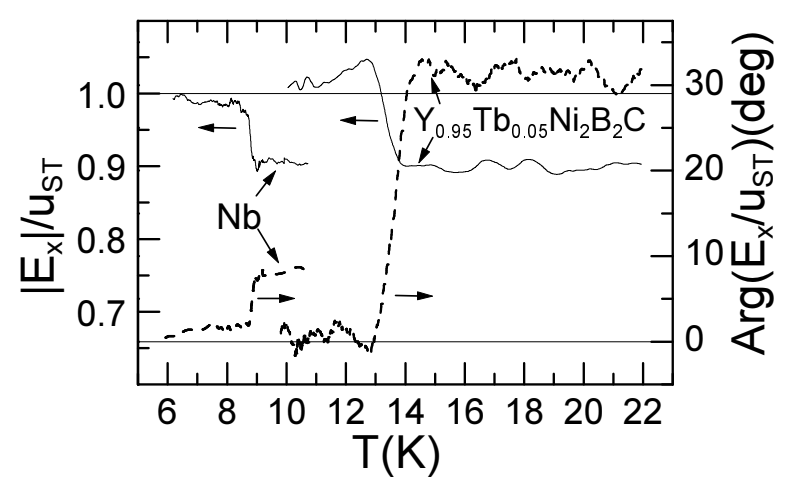

FIG. 2: The modulus (solid lines) and the phase (dashed lines) of the EF caused by the acoustic Stewart-Tolman effect $(H=0)$

frozen, and, as follows from Eqs. (3), (44), $E_{x}=u_{S T}$. It means that under such conditions the inertial force becomes the only source for the EF radiated from the sample. Therefore, it is convenient to choose the amplitude and the phase of the $E_{x}$ signal at $H=0$ and $T \ll T_{c}$ as the reference points for the complex quantity $E_{x}(H, T)$.

At $T>T_{c}$ and $H=0$ the component $E_{x}$ is given by the first term in the r.h.s. of Eq. (11). In this case it contains the contribution of the deformation force. But in rather dirty samples $\left(\left|k_{n}^{-2}\right|>\beta_{d}\right)$ the $E_{x}$ field is determined in the main part by the ST effect. In such samples the amplitude of the radiated EF should increase, while its phase should decrease under transition to the superconducting state. The experimental dependencies $\left|E_{x}(T)\right|$ and $\arg E_{x}(T)$ presented in Fig.2 demonstrate such behavior. We consider these results as the first experimental observation of this effect. We would like to emphasize that for our method the registration of the EF radiation caused by the ST effect plays the vital role, since only in this case one can obtain true amplitude and phase characteristics of the $E_{x}(H)$ dependence.

Let us mention one technical detail important for the measurement of $E_{x}$. When the receiving antenna is oriented along the weak component $E_{x}$, the measured field $E_{\text {meas }}(H)$ contains the admixture of the $E_{y}$ component caused by the edge effects: $E_{\text {meas }}(H)=$ $E_{x}(H)+\gamma E_{y}(H)$, where $\gamma$ is the complex valued geometrical factor that remains nonzero at any orientation of the antenna. The extraction of the $E_{x}(H)$ component can be done using different parity of the $E_{x}(H)$ and $E_{y}(H)$ components with respect to $H: E_{x}(H)=$ $\left[E_{\text {meas }}(H)+E_{\text {meas }}(-H)\right] / 2$. The examples of the real (in-phase with $u_{S T}$ ) and the imaginary (quadrature) parts of the $E_{x}$ field are shown in Fig. [3]

One can easily find that in the normal state the sign of the imaginary part of the second term in the r.h.s. of Eq. (11) coincides with the sign of $\Omega$. Thus, in borocarbides the carriers are of the electron type that is in agreement

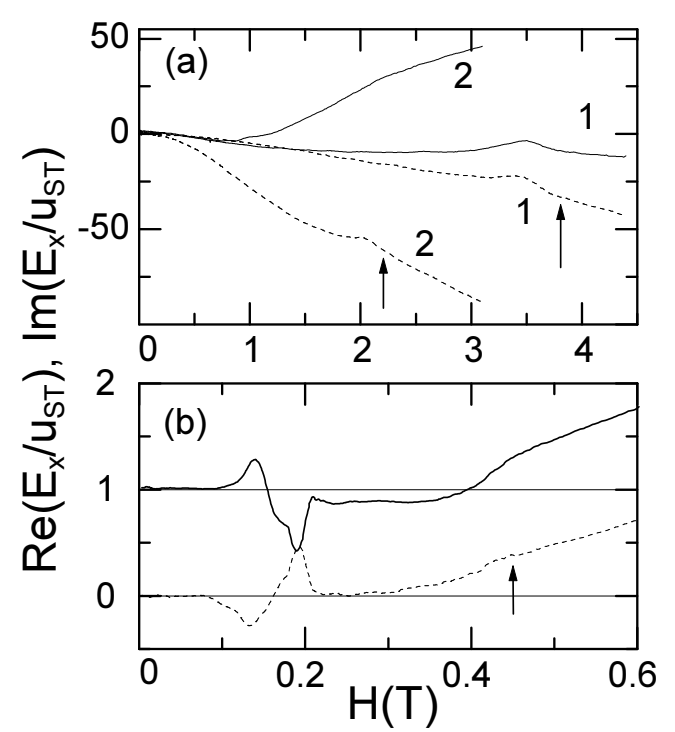

FIG. 3: The real (solid lines) and the imaginary (dotted lines) parts of $E_{x}$ for the borocarbides $\left(1-\mathrm{Y}_{0.95} \mathrm{~Tb}_{0.05} \mathrm{Ni}_{2} \mathrm{~B}_{2} \mathrm{C}\right.$ at $T=1.7 \mathrm{~K}, 2-\mathrm{LuNi}_{2} \mathrm{~B}_{2} \mathrm{C}$ at $T=9 \mathrm{~K}$ ) (a) and for $\mathrm{Nb}$ at $T=1.7 \mathrm{~K}(\mathrm{~b})$. The arrows indicate $H_{c 2}$.

with the transport data 11]. At the same time, in $\mathrm{Nb}$ the carriers are the holes (as well known, see. e.g. 12]).

Eq. (6) can be presented in the form

$\frac{E_{x}(H)}{u_{S T} X(H)}=1-i \beta_{d m}(H)-\frac{[1-X(H)]^{2}}{X(H)}\left[\frac{\omega \alpha_{M}}{q^{2} \frac{H^{2}}{4 \pi}}\right] \frac{|e| H}{m_{e} \omega c}$.

One can see that for real valued $\beta_{d m}$ and $\alpha_{M}$ the function $\alpha_{M}(H)$ can be found from the real part of Eq. (8) without the knowledge of the exact form of $\beta_{d m}(H)$.

Eq. (8) is applicable for the normal state, as well, under the replacements of the ratio $4 \pi \omega \alpha_{M} / q^{2} H^{2}$ in the r.h.s. of Eq. (8) to the quantity $\left|k_{n}^{2}\right| \Omega \tau$. To present the data in a compact form it is convenient to define formally the parameter $\omega \alpha_{M}$ at $H>H_{c 2}$ as $\omega \alpha_{M}=$ $q^{2}\left|k_{n}^{2}\right| H_{c 2}^{2} \Omega \tau / 4 \pi$.

The obtained dependencies of the Magnus coefficient on the magnetic field for the borocarbides are shown in Fig.4 One can see from Fig. (4) that for the Y-based borocarbides the dependence $\omega \alpha_{M}(H)$ is roughly linear in the whole range of the magnetic field. At $H>H_{c 2}$ one can expect the linear increase of $\omega \alpha_{M}(H)$ just from the definition of this quantity in the normal state (see above). At $H<H_{c 2}$ the linear dependence is no more than the reflection of the fact that the Magnus force is proportional to the number of vortices. In $\mathrm{LuNi}_{2} \mathrm{~B}_{2} \mathrm{C}$ the dependence $\omega \alpha_{M}(H)$ is almost saturated in the normal state. The latter is connected with a strong non-linearity of $\Omega \tau$, reported, for the first time, in Ref. 11]. We note that the coefficient $\alpha_{M}$ obtained is in two orders of magnitude smaller than $\eta$ (Ref. [10]) at the same $H$. 


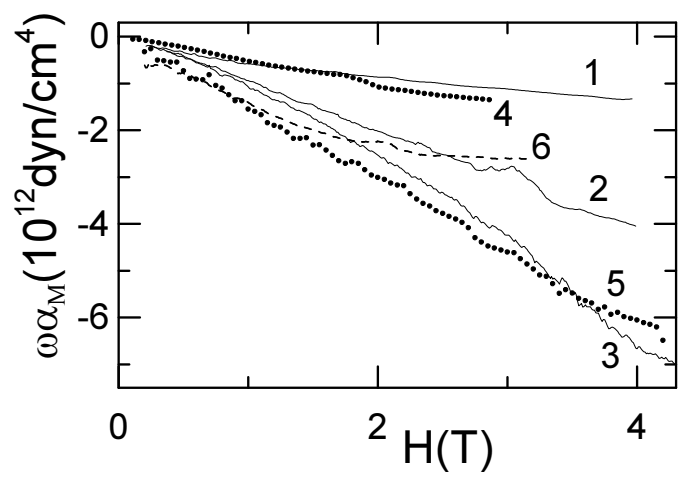

FIG. 4: Field dependencies of the Magnus coefficient. Solid lines 1,2 , and $3-\mathrm{Y}_{0.95} \mathrm{~Tb}_{0.05} \mathrm{Ni}_{2} \mathrm{~B}_{2}$ at $T=8 \mathrm{~K}\left(H_{c 2}=1.7\right.$ $\mathrm{T}) ; 4 \mathrm{~K}(3.3 \mathrm{~T})$; and $1.7 \mathrm{~K}(3.8 \mathrm{~T})$, correspondingly; dotted lines 4 and $5-\mathrm{YNi}_{2} \mathrm{~B}_{2} \mathrm{C}$ at $8 \mathrm{~K}(2 \mathrm{~T})$ and $5 \mathrm{~K}(3.8 \mathrm{~T})$, correspondingly; dashed line $6-\mathrm{LuNi}_{2} \mathrm{~B}_{2} \mathrm{C}$ at $9 \mathrm{~K}(2.15 \mathrm{~T})$.

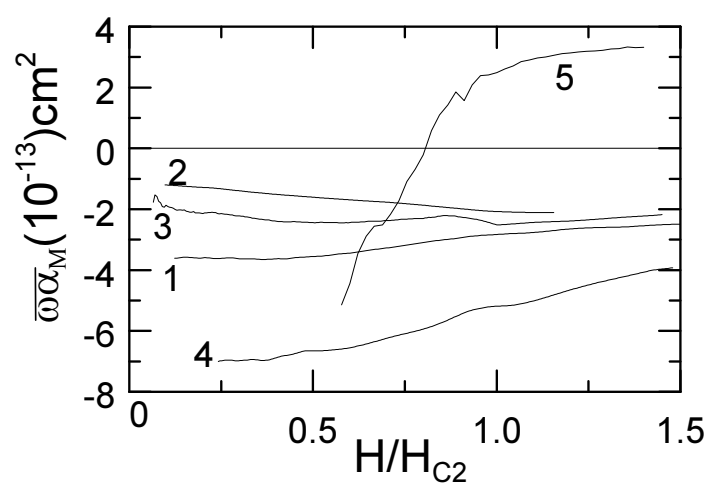

FIG. 5: The Magnus coefficient for one vortex of unit length. $1,2-\mathrm{Y}_{0.95} \mathrm{~Tb}_{0.05} \mathrm{Ni}_{2} \mathrm{~B}_{2}$ at $8 \mathrm{~K}$ and $1.7 \mathrm{~K}$, correspondingly; 3 $-\mathrm{YNi}_{2} \mathrm{~B}_{2} \mathrm{C}$ at $8 \mathrm{~K} ; 4-\mathrm{LuNi}_{2} \mathrm{~B}_{2} \mathrm{C}$ at $9 \mathrm{~K} ; 5-\mathrm{Nb}$ at $1.7 \mathrm{~K}$.

It is interesting to compare the value of the Hall coefficient $R_{H}=\Omega \tau /\left(\sigma_{0} H\right)$ that follows from our experimental data with the results obtained by the transport measurements. For the Y-based compounds at $H=4 \mathrm{~T}$ and $T=5 \mathrm{~K}$ we have $R_{H} \approx 2.8 \cdot 10^{-12} \Omega \cdot \mathrm{cm} \cdot \mathrm{Oe}^{-1}$ that almost coincides with the results of Ref. 11. For $\mathrm{LuNi}_{2} \mathrm{~B}_{2} \mathrm{C}$ at $H=4 \mathrm{~T}$ and $T=9 \mathrm{~K}$ we find $R_{H} \approx 5.3 \cdot 10^{-12}$ $\Omega \cdot \mathrm{cm} \cdot \mathrm{Oe}^{-1}$ that is also close to the value given in 11 . For $\mathrm{Nb}$ we have $R_{H} \approx 6.5 \cdot 10^{-13} \Omega \cdot \mathrm{cm} \cdot \mathrm{Oe}^{-1}$ (compare with [4]).

The quantity $\overline{\omega \alpha_{M}}=\omega \alpha_{M}\left(4 \pi / q^{2}\left|k_{n}^{2}\right|\left(H_{c 2}^{2}\right)\left(\phi_{0} / B\right)\left(\phi_{0}\right.\right.$ is the flux quantum) is shown in Fig.5 For the mixed state these data yield the Magnus coefficient for one vortex of unit length (in units of $q^{2}\left|k_{n}^{2}\right| H_{c 2}^{2} / 4 \pi$ ).

The qualitative difference in behavior of the gyroscopic force in $\mathrm{Nb}$ is apparent already from Fig. 3b. The inphase with $u_{S T}$ component of $E_{x} / u_{S T}$ becomes smaller than unity, i.e. the gyroscopic term in $E_{x}$ changes its sign. In $\mathrm{Nb}$ the parameter $\kappa$ is small and one cannot neglect the difference between $B$ and $H$. To apply the procedure of finding of $\alpha_{M}$ described above we should know the magnetic permeability $\mu(H)=B / H$. To evaluate this function we use the phenomenological expression for $\eta$ : $i \omega \eta=k_{n}^{2}\left(B H_{c 2} / 4 \pi\right)\left[1+\delta\left(1-B^{2} / H_{c 2}^{2}\right)\right]$, where $\delta$ is the fitting parameter. This expression is in better agreement with the theoretical estimates 2] and with the experiment [5] than the Bardin-Stephen formula. If the quantity $i \omega \eta$ is given, the dependencies $\mu(H)$ and $\alpha_{L}(H)$ can be determined from the experimental data. The result for $\mu(H)$ should be a smooth bounded function $\left(\left|E_{y}(H)\right| / H \leq \mu(H) \leq 1\right.$, where $E_{y}$ is in units defined above). This requirement is satisfied for $\delta \approx 0.6 \div 0.7$. The further procedure of the obtaining of $\alpha_{M}$ is the same as before. The result for $\mathrm{Nb}$ is also presented in Fig. 5 . The main qualitative conclusion is that in $\mathrm{Nb}$ the sign of the Magnus force in the mixed state is opposite to the sign of the gyroscopic force in the normal state.

In conclusion, we have used the acoustic ST effect for realization of the method of measurement of the Magnus force in type II superconductors that does not require the overthreshold flow of vortices. The measurements in non-magnetic borocarbides with conductivity of the electron type show that the gyroscopic force remains almost unchanged under the transition from the normal to the mixed state. At the same time, in $\mathrm{Nb}$ this force changes its sign below the transition point.

This study is supported in part by CRDF Foundation (Grant No UP1-2566-KH-03) and by INTAS (Grant No 03-51-3036). We would like to thank N.B.Kopnin for helpful discussions.

[1] E.B.Sonin, Phys. Rev. B 55,485 (1997).

[2] N. B. Kopnin, Rep. Progr. Phys. 65, 1633 (2002).

[3] N. B. Kopnin, V. M. Vinokur, Phys. Rev. Lett. 83, 4864 (1999).

[4] K. Noto, S.Shinzawa, Y. Muto, Sol. St. Commun. 18, 1081 (1976).

[5] V. D. Fil, D. V. Fil, Yu. A. Avramenko, A. L. Gaiduk, W. L. Johnson, Phys. Rev. B 71, 092504 (2005).

[6] M. O. Mun, S. I. Lee, W. C. Lee, P. C. Canfield, B. K. Cho, D. C. Johnston, Phys. Rev. Lett 76, 2790 (1996).

[7] E. A. Masalitin, V. D. Fil, K. N. Zhekov, A. N. Zholobenko, T. V. Ignatova, Low Temp. Phys. 29, 72 (2003).

[8] V. D. Fil, Low Temp. Phys. 27, 993 (2001).

[9] E. B. Sonin, Phys. Rev. Lett. 76, 2794 (1996).

[10] A. N. Zholobenko, G. P. Mikitik, V. D. Fil, D. V. Fil, J. D. Kim, E. M. Choi, S. I. Lee, Low. Temp. Phys., in press.

[11] V. N. Narozhnyi, J. Freudenberger, V. N. Kochetkov, K. A. Nenkov, G. Fuchs, A. Handstein, K. H. Müller, Phys. Rev B 59, 14762 (1999).

[12] A. P. Cracknell, K. C. Wong, The Fermi Surface, Clarendon Press, Oxford , 1973. 